\title{
THE IDEAL OF WEAKLY COMPACTLY GENERATED OPERATORS ACTING ON A BANACH SPACE
}

\author{
TOMASZ KANIA AND TOMASZ KOCHANEK
}

\begin{abstract}
We call a bounded linear operator acting between Banach spaces weakly compactly generated (WCG for short) if its range is contained in a weakly compactly generated subspace of its target space. This notion simultaneously generalises being weakly compact and having separable range. In a comprehensive study of the class of WCG operators, we prove that it forms a closed surjective operator ideal and investigate its relations to other classical operator ideals. By considering the $p$ th long James space $\mathcal{J}_{p}\left(\omega_{1}\right)$, we show how properties of the ideal of WCG operators (such as being the unique maximal ideal) may be used to derive results outside ideal theory. For instance, we identify the $K_{0}$-group of $\mathscr{B}\left(\mathcal{J}_{p}\left(\omega_{1}\right)\right)$ as the additive group of integers.
\end{abstract}

\section{INTRODUCTION}

Amir and Lindenstrauss [2] initiated the study of weakly compactly generated (WCG for short) Banach spaces, that is, Banach spaces containing a weakly compact fundamental subset. Any reflexive and any separable Banach space is weakly compactly generated. Other notable examples include $L_{1}(\mu)$-spaces with a $\sigma$-finite positive measure $\mu$, and $c_{0}(\Gamma)$ spaces for an arbitrary index set $\Gamma$. The latter play a special role, as for every WCG space $X$ there is a bounded linear operator which maps $X$ injectively into $c_{0}(\Gamma)$ for some $\Gamma$. On the other hand, there are plenty of Banach spaces which are not WCG such as $\ell_{\infty}$ and $\ell_{1}(\Gamma)$ for any uncountable index set $\Gamma$.

According to Lindenstrauss [26], the class of WCG Banach spaces is stable under quotients, $c_{0}$-sums, $\ell_{p}$-sums for $p \in(1, \infty)$, and countable $\ell_{1}$-sums. Surprisingly, a closed subspace of a WCG Banach space need not be WCG. The first counterexample was given by Rosenthal [30] who exhibited a non-WCG subspace of $L_{1}(\mu)$ for some probability measure $\mu$. Note that the aforementioned spaces $\ell_{\infty}$ and $\ell_{1}(\Gamma)$ (with $\Gamma$ uncountable) are not subspaces of any WCG space. We refer to [3] for further examples concerning that subspace problem, and to [15] for a list of necessary and sufficient conditions for being a (subspace of a) WCG Banach space.

Let $T: X \rightarrow Y$ be a bounded linear operator acting between Banach spaces $X$ and $Y$. We call the operator $T$ weakly compactly generated (or WCG) if there is a WCG subspace $Z$ of $Y$ such that $T(X) \subseteq Z$. We shall prove in Section 2 (Theorem 2.1) that the class $\mathscr{W} \mathscr{C} \mathscr{G}$ of all weakly compactly generated operators forms a closed operator ideal. Moreover, this operator ideal is a surjective, but neither it is injective nor symmetric (Propositions 2.3

2000 Mathematics Subject Classification. Primary 47L10, 47L20; Secondary 46H10, 46B26.

Key words and phrases. operator ideal, weakly compactly generated, WCG space. 
and 2.4). We then compare $\mathscr{W} \mathscr{C} \mathscr{G}$ to other classical operator ideals, including operator ideals of weakly compact, completely continuous, strictly singular and strictly cosingular operators.

Section 3 is devoted to weakly compactly generated operators acting on the $p$ th long James space $\mathcal{J}_{p}\left(\omega_{1}\right)(p \in(1, \infty))$. The main result of this section (Theorem 3.7) asserts that the ideal of weakly compactly generated operators is the unique maximal ideal of the algebra $\mathscr{B}\left(\mathcal{J}_{p}\left(\omega_{1}\right)\right)$ of bounded operators on $\mathcal{J}_{p}\left(\omega_{1}\right)$. Buliding on the techniques from [17], some further descriptions of this ideal are given, and these lead to additional results concerning commutators, automatic continuity of homomorphisms and the $K_{0}$-group of $\mathscr{B}\left(\mathcal{J}_{p}\left(\omega_{1}\right)\right)$.

Next we turn our attention to operators acting on $C(K)$-spaces. In terms of the representing measure of a given operator $T: C(K) \rightarrow X$, we give a sufficient condition for $T$ being WCG (Theorem 4.2). This is an application of the characterisation of subspaces of WCG Banach spaces, obtained by Fabian, Montesinos and Zizler [13.

Finally, in Section 5 we discuss some examples of non-Eberlein compacta $K$ for which the ideal of WCG operators on $C(K)$ is maximal. In particular we show that this is the case for a certain Mrówka space $K$ constructed by Koszmider [19], and we give a complete description of the lattice of closed ideals in $\mathscr{B}(C(K))$ (Theorem 5.5).

Throughout this paper, Banach spaces are assumed to be over the field $\mathbb{K}=\mathbb{R}$ or $\mathbb{K}=\mathbb{C}$, unless the field is explicitly specified. By an operator we understand a bounded linear operator acting between Banach spaces. An operator $T: E \rightarrow F$ is bounded below if there exists a constant $\gamma>0$ such that $\|T x\| \geqslant \gamma\|x\|$ for every $x \in E$, which means that $T$ is one-to-one and has closed range. The space $\mathscr{B}(E, F)$ of all operators $T: E \rightarrow F$ is a Banach space, when endowed with the operator norm and $\mathscr{B}(E, E)=\mathscr{B}(E)$ is a unital Banach algebra with multiplication being composition of operators.

Let $\mathscr{B}$ be the class of all operators acting between arbitrary Banach spaces. By an operator ideal we understand a subclass $\mathscr{J}$ of $\mathscr{B}$, containing the identity operator on the one-dimensional Banach space and which assigns to each pair $(E, F)$ of Banach spaces a (not necessarily closed) linear subspace $\mathscr{J}(E, F)=\mathscr{B}(E, F) \cap \mathscr{J}$ such that for any Banach spaces $X, Y, E, F$ and for any operators $T \in \mathscr{B}(X, E), S \in \mathscr{J}(E, F)$ and $R \in \mathscr{B}(F, Y)$ we have $R S T \in \mathscr{J}(X, Y)$. An operator ideal $\mathscr{J}$ is closed, if the subspace $\mathscr{J}(E, F)$ is closed in $\mathscr{B}(E, F)$ for any pair $(E, F)$ of Banach spaces. We refer to [29] for the general theory of operator ideals.

The classes $\mathscr{K}, \mathscr{W}$ and $\mathscr{X}$ of compact operators, weakly compact operators and operators with separable range are standard examples of closed operator ideals, respectively.

For any Banach space $X$ the class $\mathscr{G}_{X} \subseteq \mathscr{B}$ which assigns to each pair $(E, F)$ of Banach spaces the subspace

$$
\mathscr{G}_{X}(E, F)=\operatorname{span}\{S T: T \in \mathscr{B}(E, X), S \in \mathscr{B}(X, F)\} \subseteq \mathscr{B}(E, F)
$$

is the ideal of operators factoring through $X$. In the case where $X$ contains a complemented copy of its Cartesian square $X \oplus X$, the set $\{S T: T \in \mathscr{B}(E, X), S \in \mathscr{B}(X, F)\}$ is itself a linear subspace of $\mathscr{B}(E, F)$, whence the symbol 'span' above can be suppressed. 
2. THE OPERATOR IDEAL OF WEAKLY COMPACTLY GENERATED OPERATORS AND ITS RELATIONS TO OTHER OPERATOR IDEALS

Recall that $\mathscr{W} \mathscr{C} \mathscr{G}(E, F)$ denotes the set of all operators $T: E \rightarrow F$ with $T(E)$ contained in a weakly compactly generated subspace of $F$.

Theorem 2.1. The class $\mathscr{W} \mathscr{C G}$ is a closed operator ideal.

Proof. Let $E$ and $F$ be Banach spaces. Fix two operators $T$ and $S$ in $\mathscr{W} \mathscr{C} \mathscr{G}(E, F)$. We deduce that $T+S$ belongs to $\mathscr{W} \mathscr{C} \mathscr{G}(E, F)$. Indeed, let $K_{T}$ and $K_{S}$ be two weakly compact subsets of $E$ such that $T(E) \subseteq \overline{\operatorname{span}} K_{T}$ and $S(E) \subseteq \overline{\operatorname{span}} K_{S}$. The union $K_{T} \cup K_{S}$ is weakly compact and $(T+S)(E) \subseteq \overline{\operatorname{span}}\left(K_{T} \cup K_{S}\right)$, hence $T+S \in \mathscr{W} \mathscr{C} \mathscr{G}(E, F)$.

Let $X, Y, E, F$ be Banach spaces and let $T \in \mathscr{B}(X, E), S \in \mathscr{W} \mathscr{C G}(E, Y)$ and $R \in$ $\mathscr{B}(Y, F)$. We note that both $S T$ and $R S$ are in the class $\mathscr{W} \mathscr{C} \mathscr{G}$. Indeed, since $S(T(X)) \subseteq$ $S(E)$ and $S(E)$ is a subspace of a WCG subspace of $Y$ we have $S T \in \mathscr{W} \mathscr{C} \mathscr{G}(X, Y)$. Now, let $K$ be a weakly compact subset of $Y$ such that $S(E) \subseteq \overline{\operatorname{span}} K$. Every operator is weakto-weak continuous, thus the image $R(K)$ is weakly compact and $R S(E) \subseteq R(\overline{\operatorname{span}} K) \subseteq$ $\overline{\text { span }} R(K)$. Consequently, $R S \in \mathscr{W} \mathscr{C} \mathscr{G}(X, Y)$.

Finally, we shall prove that $\mathscr{W} \mathscr{C} \mathscr{G}$ is closed. Let $\left(T_{n}\right)_{n=1}^{\infty} \subseteq \mathscr{W} \mathscr{C} \mathscr{G}(E, F)$ be a normconvergent sequence of operators with limit $T$, say. Let $F_{n}$ be a WCG subspace of $F$ such that $T_{n}(E) \subseteq F_{n}(n \in \mathbb{N})$. Define $W$ to be the $\ell_{1}$-sum of $\left(F_{n}\right)_{n=1}^{\infty}$, which is again a WCG Banach space [26, Proposition 2.4]. Furthermore, let $J: W \rightarrow F$ be the operator defined by $J\left(x_{n}\right)_{n=1}^{\infty}=\sum_{n=1}^{\infty} x_{n}$. Since $W$ is WCG, the space $\overline{J(W)}$ is WCG as well, and we have $T(E) \subseteq \overline{\operatorname{span}} \bigcup_{n \in \mathbb{N}} F_{n} \subseteq \overline{J(W)}$.

Proposition 2.2. The ideal $\mathscr{W} \mathscr{C G}(E)$ is a proper ideal of $\mathscr{B}(E)$ if and only if $E$ is not weakly compactly generated.

Proof. This follows by considering the range of the identity operator on $E$.

Let $E, E_{0}$ and $F$ be arbitrary Banach spaces. Recall that an operator ideal $\mathscr{J}$ is surjective if for any surjective operator $Q \in \mathscr{B}\left(E, E_{0}\right)$ and each operator $T \in \mathscr{B}\left(E_{0}, F\right)$, we have $T \in \mathscr{J}\left(E_{0}, F\right)$ provided $T Q \in \mathscr{J}(E, F)$. An operator ideal $\mathscr{J}$ is injective if for each closed subspace $F_{0}$ of $F$ and every operator $T \in \mathscr{B}\left(E, F_{0}\right)$ with $\iota T \in \mathscr{J}(E, F)$ we have $T \in \mathscr{J}\left(E, F_{0}\right)$ (here $\iota: F_{0} \rightarrow F$ denotes the canonical embedding).

Proposition 2.3. The operator ideal $\mathscr{W} \mathscr{C G}$ is surjective, but not injective.

Proof. For the surjectivity of $\mathscr{W} \mathscr{C} \mathscr{G}$, suppose that $T \in \mathscr{B}(E, F)$ satisfies $T Q \in \mathscr{W} \mathscr{C} \mathscr{G}\left(E_{0}, E\right)$ for some Banach space $E_{0}$ and some surjection $Q \in \mathscr{B}\left(E_{0}, E\right)$. Since $Q$ is a surjection, the ranges of $T Q$ and $T$ are the same, hence $T \in \mathscr{W} \mathscr{C} \mathscr{G}(E, F)$.

We observe that $\mathscr{W} \mathscr{C} \mathscr{G}$ is not injective. Indeed, it follows from the existence of closed subspace of a WCG space which is not WCG [30].

Proposition 2.4. The operator ideal $\mathscr{W} \mathscr{C G}$ is not symmetric, that is, the adjoint of a weakly compactly generated operator need not be weakly compactly generated.

Conversely, if the adjoint of an operator $T$ is weakly compactly generated, then again, $T$ need not be. 
Proof. The lack of symmetry is clear - the identity operator on $\ell_{1}$ is WCG, while its adjoint $I_{\ell_{1}}^{*}=I_{\ell_{\infty}}$ is not.

Now, let $J L$ be the Johnson-Lindenstrauss space (consult [16] for its definition and properties), which is known not to be a WCG space but its dual $J L^{*}=\ell_{1} \oplus \ell_{2}(\mathfrak{c})$ clearly is. Take $T$ to be the identity on $J L$. Then, $T=I_{J L}$ is not WCG, whereas $T^{*}=I_{J L^{*}}$ is.

Let us observe that each operator which factors through a WCG space is weakly compactly generated. In particular, one can deduce from this that weakly compact operators are weakly compactly generated. The Davis-Figiel-Johnson-Pełczyński theorem [10, Theorem 6.2.15] characterises weakly compact operators as precisely those which admit a factorisation through a reflexive space, hence each weakly compact operator is WCG; of course such a heavy machinery is superfluous in this case as it can be seen directly. Trivially, operators with separable range are WCG as well.

In the remaining part of this section we shall study order relations between $\mathscr{W} \mathscr{C} \mathscr{G}$ and some classical operator ideals. Recall that an operator $T \in \mathscr{B}(E, F)$ is

(a) completely continuous (or Dunford-Pettis) if, it maps weakly convergent sequences in $E$ to norm convergent sequences in $F$;

(b) strictly singular (or Kato) if, it is not bounded below when restricted to any closed, infinite-dimensional subspace of its domain;

(c) strictly cosingular (or Petczyński) if, for each infinite-codimensional, closed subspace $M$ of $F$, the operator $\pi T$ is not surjective, where $\pi: F \rightarrow F / M$ is the quotient operator.

\section{Proposition 2.5.}

(i) The operator ideal $\mathscr{W}$ is a proper subclass of $\mathscr{W} \mathscr{C} \mathscr{G}$.

(ii) The operator ideals $\mathscr{V}, \mathscr{S}$ and $\mathscr{C} \mathscr{S}$ are incomparable to $\mathscr{W} \mathscr{C} \mathscr{G}$.

Proof. (i) This is clear as explained above.

(ii) To see that $\mathscr{V} \nsubseteq \mathscr{W} \mathscr{C} \mathscr{G}$, recall that any operator on $\ell_{1}\left(\omega_{1}\right)$ is completely continuous, so that the identity on $\ell_{1}\left(\omega_{1}\right)$ belongs to $\mathscr{V}\left(\ell_{1}\left(\omega_{1}\right)\right) \backslash \mathscr{W} \mathscr{C} \mathscr{G}\left(\ell_{1}\left(\omega_{1}\right)\right)$.

Conversely, to prove that $\mathscr{W} \mathscr{C} \mathscr{G} \nsubseteq \mathscr{V}$, by the Rosenthal-Dor $\ell_{1}$-theorem [9, Chapter XI] any completely continuous operator on a space without a copy of $\ell_{1}$ is compact. Thus, the identity operator on any infinite-dimensional WCG Banach space without a subspace isomorphic to $\ell_{1}$ belongs to $\mathscr{W} \mathscr{C} \mathscr{G} \backslash \mathscr{V}$.

The relations $\mathscr{W} \mathscr{C} \mathscr{G} \nsubseteq \mathscr{S}, \mathscr{W} \mathscr{C} \mathscr{G} \nsubseteq \mathscr{C} \mathscr{S}$ are clear, simply consider the identity operator on an infinite-dimensional WCG Banach space.

Let $T: C\left[0, \omega_{1}\right] \rightarrow \ell_{\infty}\left(\left[0, \omega_{1}\right]\right)$ be the inclusion operator. Since $C\left[0, \omega_{1}\right]$ is not WCG, the operator $T$ is not in $\mathscr{W} \mathscr{C} \mathscr{G}$. To prove that $\mathscr{W} \mathscr{C} \mathscr{G} \nsupseteq \mathscr{C} \mathscr{S}$, it is enough to show that $T$ is strictly cosingular. In the light of a result by Bourgain and Diestel [6], it suffices to ensure that $T^{*}$ maps weak ${ }^{*}$-null sequences into norm-null sequences. This is, however, automatic since weak ${ }^{*}$-null sequences in $\ell_{\infty}\left(\left[0, \omega_{1}\right]\right)^{*}$ converge weakly (this is the counterpart of $[9$, Theorem VII.15] for uncountable index sets), $T^{*}$ is weak-to-weak continuous like every operator and $\ell_{1}\left(\left[0, \omega_{1}\right]\right) \cong C\left[0, \omega_{1}\right]^{*}$ has the Schur property, which means that weakly convergent sequences in $C\left[0, \omega_{1}\right]^{*}$ converge in norm. 
For the relation $\mathscr{W} \mathscr{C} \mathscr{G} \nsupseteq \mathscr{S}$, take a set $\Gamma$ such that there is a surjective operator $T: \ell_{1}(\Gamma) \rightarrow C\left[0, \omega_{1}\right]$. We have $T \notin \mathscr{W} \mathscr{C} \mathscr{G}$, because $C\left[0, \omega_{1}\right]$ is not weakly compactly generated. To see that $T$ is strictly singular, observe that if $\left.T\right|_{X}$ was bounded below on some infinite-dimensional subspace $X$ of $\ell_{1}(\Gamma)$, one could find an isomorphic copy $Y$ of $c_{0}$ in $T(X)$ (as $C\left[0, \omega_{1}\right]$ is $c_{0}$-saturated, that is, each infinite-dimensional, closed subspace of $C\left[0, \omega_{1}\right]$ contains a subspace isomorphic to $c_{0}$; cf. also [25]). Consequently, $\left(\left.T\right|_{X}\right)^{-1}(Y)$ would be an isomorphic copy of $c_{0}$ in $\ell_{1}(\Gamma)$, which is impossible.

\section{WeAkly COMPACTLY Generated operators on the LONG JAMES SPACE}

In this section we prove that the ideal of weakly compactly generated operators is the unique maximal ideal of the algebra of bounded operators on the $p$ th long James space $\mathcal{J}_{p}\left(\omega_{1}\right)$. Then we derive from this fact several characterisations of this ideal. The long James space (originally for $p=2$ ) serves as a counterexample to numerous questions in Banach space theory (consult Edgar's paper [12] for details).

Let $p \in(1, \infty)$. For any ordinal $\eta$ and any function $x:[0, \eta) \rightarrow \mathbb{K}$ define

$$
\|x\|_{p, 0}=\sup \left\{\left(\sum_{j=1}^{n}\left|x\left(\alpha_{j}\right)-x\left(\alpha_{j-1}\right)\right|^{p}\right)^{1 / p}: n \in \mathbb{N} \text { and } 0 \leqslant \alpha_{0}<\alpha_{1}<\ldots<\alpha_{n}<\eta\right\} .
$$

Edgar [12] defined the long James space to be

$$
\mathcal{J}_{p}^{(0)}(\eta)=\left\{x:[0, \eta) \rightarrow \mathbb{K} \mid x \text { is continuous, } x(0)=0 \text { and }\|x\|_{p, 0}<\infty\right\} .
$$

In fact, for our purposes we require a slight modification of Edgar's construction. Let $\eta$ be a non-zero limit ordinal. We set

$$
\widetilde{\mathcal{J}}_{p}(\eta)=\left\{x:[0, \eta) \rightarrow \mathbb{K} \mid \lim _{\alpha \rightarrow \eta} x(\alpha)=0 \text { and }\|x\|_{p, 0}<\infty\right\}
$$

and define $\mathcal{J}_{p}(\eta)=\left\{x \in \widetilde{\mathcal{J}}_{p}(\eta): x\right.$ is continuous $\}$. It turns out that all these three spaces are pairwise isomorphic. Indeed, the unique order preserving bijection $\varphi$, from $\eta$ onto the set $D(\eta)$ of all successors less than $\eta$, induces an isometry $U: \widetilde{\mathcal{J}}_{p}(\eta) \rightarrow \mathcal{J}_{p}(\eta)$ via the formula

$$
U(x)(\alpha)= \begin{cases}x\left(\varphi^{-1}(\alpha)\right), & \text { if } \alpha \in D(\eta), \\ \lim _{\beta \rightarrow \alpha} x(\beta), & \text { if } \alpha \in \eta \backslash D(\eta),\end{cases}
$$

whereas the map $V: \mathcal{J}_{p}(\eta) \rightarrow \mathcal{J}_{p}^{(0)}(\eta)$, given by

$$
V(x)(\alpha)= \begin{cases}0, & \text { if } \alpha=0 \\ x(0)+x(\alpha), & \text { if } 0<\alpha<\eta \\ x(0), & \text { if } \alpha=\eta\end{cases}
$$

yields an isomorphism between $\mathcal{J}_{p}(\eta)$ and $\mathcal{J}_{p}^{(0)}(\eta)$.

All these spaces may also be equipped with the norm

$$
\begin{array}{r}
\|x\|_{\mathcal{J}_{p}}=2^{-1 / p} \sup \left\{\left(\left|x\left(\alpha_{n}\right)-x\left(\alpha_{0}\right)\right|^{p}+\sum_{j=1}^{n}\left|x\left(\alpha_{j}\right)-x\left(\alpha_{j-1}\right)\right|^{p}\right)^{1 / p}: n \in \mathbb{N}\right. \text { and } \\
\left.0 \leqslant \alpha_{0}<\alpha_{1}<\ldots<\alpha_{n}<\eta\right\},
\end{array}
$$


which is more natural than $\|\cdot\|_{p, 0}$ in the sense that $\left\|e_{\alpha}\right\|_{\mathcal{J}_{p}}=1$ for every $\alpha<\eta$, where $e_{\alpha}=\mathbf{1}_{\{\alpha\}}$. Moreover, for any $x \in \widetilde{\mathcal{J}}_{p}(\eta)$ we have

$$
2^{-1 / p}\|x\|_{p, 0} \leqslant\|x\|_{\mathcal{J}_{p}} \leqslant 2^{1 / p}\|x\|_{p, 0}
$$

According to [12, Propositions 1, 3], we know that

- $\left(\mathbf{1}_{(\alpha, \eta]}\right)_{0 \leqslant \alpha<\eta}$ is a basis for $\mathcal{J}_{p}^{(0)}(\eta)$;

- $\left(e_{\alpha}^{*}\right)_{0<\alpha \leqslant \eta}$ is a basis for $\mathcal{J}_{p}^{(0)}(\eta)^{*}$,

where $e_{\alpha}^{*}(f)=f(\alpha)\left(\alpha<\eta, f \in \mathcal{J}_{p}^{(0)}(\eta)\right)$. By applying the isomorphism $V$ and the dual isomorphism $V^{*}$, we infer that

- $\left(\mathbf{1}_{[0, \alpha]}\right)_{0 \leqslant \alpha<\eta}$ is a basis for $\mathcal{J}_{p}(\eta)$;

- $\left(e_{\alpha}^{*}\right)_{0 \leqslant \alpha<\eta}$ is a basis for $\mathcal{J}_{p}(\eta)^{*}$.

From now, we specialise to $\eta=\omega_{1}$, the smallest uncountable ordinal. Let us recall that $\mathcal{J}_{p}\left(\omega_{1}\right)$ is isomorphic to its bidual and has the Radon-Nikodým property, yet it is not isomorphic to a subspace of a WCG Banach space.

In the case of the classical James space $\mathcal{J}_{p}$ it was shown by Laustsen that $\mathscr{W}\left(\mathcal{J}_{p}\right)$ is the unique maximal ideal of $\mathscr{B}\left(\mathcal{J}_{p}\right)$ and, moreover, that an operator on $\mathcal{J}_{p}$ is weakly compact if and only if it factors through the reflexive space $\left(\bigoplus_{n=1}^{\infty} \mathcal{J}_{p}(n)\right)_{\ell_{p}}$, where $\mathcal{J}_{p}(n)=$ $\operatorname{span}\left\{e_{j}\right\}_{j \leqslant n}$ (see [21, Theorem 4.16] and [22, Theorem 4.3], respectively). According to Willis (cf. [31, Proposition 6]), the ideal $\mathscr{W}\left(\mathcal{J}_{p}\right)$ may be also characterised as the ideal of compressible operators. Let us recall that for any Banach space $X$ an operator $T \in \mathscr{B}(X)$ is said to be compressible if there is $n \in \mathbb{N}$, and a sequence $\left(Q_{k}\right)_{k=1}^{\infty}$ of projections on $X^{n}$, such that $Q_{k} Q_{\ell}=0$ whenever $k \neq \ell$ and $T$ factors through $Q_{k}$ for each $k \in \mathbb{N}$. Equivalently (cf. [31, Proposition 1]), $T \in \mathscr{B}(X)$ is compressible if and only if there exist $n \in \mathbb{N}$ and sequences $\left(D_{k}\right)_{k=1}^{\infty}$ and $\left(E_{k}\right)_{k=1}^{\infty}$ of closed subspaces of $X^{n}$ such that

(c1) $X^{n}=D_{1} \oplus E_{1}$ and $E_{k}=D_{k+1} \oplus E_{k+1}$ for every $k \in \mathbb{N}$;

(c2) $T$ factors through $D_{k}$ for every $k \in \mathbb{N}$.

We denote by $\mathscr{C}(X)$ the ideal of compressible operators on $X$.

The methods used by Laustsen and Willis to obtain characterisations of the ideal $\mathscr{W}\left(\mathcal{J}_{p}\right)$ are based on Lemma 2.1 from the paper [24] by Loy and Willis. It asserts that every operator $T \in \mathscr{W}\left(\mathcal{J}_{p}\right)$ admits a decomposition $T=K+R$, where $K$ is compact and $R$ has only finitely many non-zero entries in each line of its matrix representation, and also satisfies $R^{* *}\left(\mathbf{1}_{\mathbb{N}}\right)=0$. This approach is rather useless for characterising the ideal $\mathscr{W} \mathscr{C} \mathscr{G}\left(\mathcal{J}_{p}\left(\omega_{1}\right)\right)$, since weakly compactly generated operators are not characterised by properties of their second adjoints, unlike weakly compact operators.

Our approach is based on Lemma 1.2 from [17] by the first-named author, Koszmider and Laustsen, which refines certain results of Alspach and Benyamini from [1]. We shall prove a counterpart of that lemma for $\mathcal{J}_{p}\left(\omega_{1}\right)$. Before proceeding to the proof let us note that, in view of the theorem of Hagler and Johnson [14, Corollary 2], and the fact that $\mathcal{J}_{p}\left(\omega_{1}\right)^{*}$ has the Radon-Nikodým property, the unit ball of $\mathcal{J}_{p}\left(\omega_{1}\right)^{*}$ is weak ${ }^{*}$ sequentially compact. Though the general idea of the proof remains the same, some modification is 
needed, as the original argument heavily relies on the identification $C[0, \alpha]^{*} \cong \ell_{1}([0, \alpha])$ for any ordinal $\alpha$.

Theorem 3.1. For every $p \in(1, \infty)$ and every $T \in \mathscr{B}\left(\mathcal{J}_{p}\left(\omega_{1}\right)\right)$ there exists a $\lambda \in \mathbb{K}$ such that for some club set (a closed and unbounded set) $D \subseteq \omega_{1}$ we have

$$
e_{\alpha}^{*} T(x)=\lambda e_{\alpha}^{*} x \quad \text { for } x \in \mathcal{J}\left(\omega_{1}\right) \text { and } \alpha \in D .
$$

Proof. For each $\alpha<\omega_{1}$ define $\varphi_{\alpha}=T^{*} e_{\alpha}^{*}$. We shall prove that for some $\lambda \in \mathbb{K}$ there is a club subset $D \subset \omega_{1}$ with $\varphi_{\alpha}=\lambda e_{\alpha}^{*}$, since then $\left\langle T x, e_{\alpha}^{*}\right\rangle=\left\langle x, T^{*} e_{\alpha}^{*}\right\rangle=\lambda e_{\alpha}^{*} x$ for $x \in X$, $\alpha \in D$, and the assertion would follow.

Let $\mathcal{Q}$ be any countable, dense subset of $\mathbb{K}$. For every $\alpha<\omega_{1}$ and $k \in \mathbb{N}$ we may find a finite set $F_{\alpha, k} \subset \omega_{1}$ and scalars $q_{\alpha, k, \beta} \in \mathcal{Q}$ (for $\beta \in F_{\alpha, k}$ ) such that

$$
\left\|\varphi_{\alpha, k}-\varphi_{\alpha}\right\|<1 / k, \quad \text { where } \quad \varphi_{\alpha, k}=\sum_{\beta \in F_{\alpha, k}} q_{\alpha, k, \beta} e_{\beta}^{*} \text {. }
$$

CLAIM 1. If $\left(\alpha_{k}\right)_{k=1}^{\infty} \subset \omega_{1}$ and $\alpha_{k} \rightarrow \alpha \in \omega_{1}$, then $\varphi_{\alpha_{k}, k} \stackrel{w *}{\longrightarrow} \varphi_{\alpha}$.

Proof of Claim 1. For any $x \in \mathcal{J}_{p}\left(\omega_{1}\right)$ we have

$$
\left|\left(\varphi_{\alpha_{k}, k}-\varphi_{\alpha}\right)(x)\right| \leqslant\left\|\varphi_{\alpha_{k}, k}-\varphi_{\alpha_{k}}\right\| \cdot\|x\|_{\mathcal{J}_{p}}+\left|\left(\varphi_{\alpha_{k}}-\varphi_{\alpha}\right)(x)\right| .
$$

The first term tends to zero by (3.2). By the continuity of each $x \in \mathcal{J}_{p}\left(\omega_{1}\right)$, we have $e_{\alpha_{k}}^{*} \stackrel{w *}{\longrightarrow} e_{\alpha}^{*}$, thus the weak ${ }^{*}$-continuity of $T^{*}$ gives $\varphi_{\alpha_{k}}=T^{*} e_{\alpha_{k}}^{*} \stackrel{w *}{\longrightarrow} T^{*} e_{\alpha}^{*}=\varphi_{\alpha}$, which means that the second term tends to zero as well.

For each $k \in \mathbb{N}$ the $\Delta$-system lemma produces an uncountable set $A_{k} \subset \omega_{1}$ and a root $\Delta_{k}=\left\{\beta_{k, 1}, \ldots, \beta_{k,\left|\Delta_{k}\right|}\right\} \subset \omega_{1}$ such that

$$
F_{\alpha, k} \cap F_{\alpha^{\prime}, k}=\Delta_{k} \quad \text { for } \alpha, \alpha^{\prime} \in A_{k}, \alpha \neq \alpha^{\prime} .
$$

By deleting at most countably many elements from each of $A_{k}$ 's we may also assume that:

(a) for every $k \in \mathbb{N}$ there is an $m_{k} \in \mathbb{N}$ with $\left|F_{\alpha, k}\right|=m_{k}$ for each $\alpha \in A_{k}$;

(b) $\sup \bigcup_{i \in \mathbb{N}} \Delta_{i}<\min \left(F_{\alpha, k} \backslash \Delta_{k}\right)$ for every $k \in \mathbb{N}$ and $\alpha \in A_{k}$;

(c) for every $k \in \mathbb{N}$ and $\alpha \in A_{k}$ there is an order preserving bijection $\sigma_{\alpha, k}:\left[m_{k}\right] \rightarrow F_{\alpha, k}$ such that $\sigma_{\alpha, k}(i)=\beta_{k, i}$ for each $1 \leqslant i \leqslant\left|\Delta_{k}\right|$

(d) for every $k \in \mathbb{N}$ there are scalars $q_{k, i} \in \mathcal{Q}$ (for $1 \leqslant i \leqslant m_{k}$ ) such that $q_{\alpha, k, \sigma_{\alpha, k}(i)}=q_{k, i}$ for $\alpha \in A_{k}$ and $1 \leqslant i \leqslant m_{k}$.

Case 1. $m_{k}=\left|\Delta_{k}\right|$ for infinitely many $k$ 's.

Without loss of generality, we may suppose that $m_{k}=\left|\Delta_{k}\right|$ for every $k \in \mathbb{N}$. Then for every $k \in \mathbb{N}$ and $\alpha \in A_{k}$ we have $F_{\alpha, k}=\Delta_{k}$ and

$$
\varphi_{\alpha, k}=\rho_{k}:=\sum_{1 \leqslant i \leqslant\left|\Delta_{k}\right|} q_{k, i} e_{\beta_{k, i}}^{*} .
$$

We define the club $D \subset \omega_{1}$ as the set of all limits of sequences $\left(\alpha_{k}\right)_{k=1}^{\infty}$ with $\alpha_{k} \in A_{k}$ for $k \in \mathbb{N}$. Now, if $\alpha \in D$ is the limit of such a sequence, then Claim 1 implies $\rho_{k}=$ $\varphi_{\alpha_{k}, k} \stackrel{w *}{\longrightarrow} \varphi_{\alpha}$. Consequently, for all $\alpha \in D$ the functional $\varphi_{\alpha}$ is the same, and is equal to 
the weak* limit of $\left(\rho_{k}\right)_{k \in \mathbb{N}}$. Moreover, since each $x \in \mathcal{J}_{p}\left(\omega_{1}\right)$ satisfies $\lim _{\alpha \rightarrow \omega_{1}} x(\alpha)=0$, we

have $\varphi_{\alpha}=T^{*} e_{\alpha}^{*} \underset{\alpha \rightarrow \omega_{1}}{\stackrel{w *}{\longrightarrow}} T^{*}(0)=0$, thus $\varphi_{\alpha}=0$ for $\alpha \in D$ and our assertion is valid with $\lambda=0$.

Case 2. $m_{k}>\left|\Delta_{k}\right|$ for infinitely many $k$ 's.

With no loss of generality we may suppose that $m_{k}>\left|\Delta_{k}\right|$ for every $k \in \mathbb{N}$. For each $k \in \mathbb{N}$ and $\alpha \in A_{k}$ define

$$
\psi_{\alpha, k}=\sum_{\left|\Delta_{k}\right|<j \leqslant m_{k}} q_{k, j} e_{\sigma_{\alpha, k}(j)}^{*}
$$

that is, $\varphi_{\alpha, k}=\rho_{k}+\psi_{\alpha, k}$. For every $k \in \mathbb{N}$ and $\alpha<\omega_{1}$ we have $\left\|\varphi_{\alpha, k}\right\| \leqslant 1 / k+\left\|T^{*} e_{\alpha}^{*}\right\| \leqslant$ $1+\|T\|$. Since the unit dual ball of $\mathcal{J}_{p}\left(\omega_{1}\right)$ is weak* sequentially compact, we may find a strictly increasing sequence $\left(k_{i}\right)_{i=1}^{\infty}$ of natural numbers, and a strictly increasing sequence $\left(\beta_{i}\right)_{i=1}^{\infty} \subset \omega_{1}$ such that $\beta_{i} \in A_{k_{i}}$ for each $i \in \mathbb{N}$ and $\varphi_{\beta_{i}, k_{i}} \stackrel{w *}{\longrightarrow} \varphi_{0}$ for some $\varphi_{0} \in \mathcal{J}_{p}\left(\omega_{1}\right)^{*}$.

CLAIM 2. There exist a number $\lambda \in \mathbb{K}$ and a functional $\rho \in \mathcal{J}_{p}\left(\omega_{1}\right)^{*}$ such that

$$
\sum_{\left|\Delta_{k_{i}}\right|<j \leqslant m_{k_{i}}} q_{k_{i}, j} \underset{i \rightarrow \infty}{\longrightarrow} \lambda \text { and } \rho_{k_{i}} \underset{i \rightarrow \infty}{\stackrel{w *}{\longrightarrow}} \rho .
$$

Proof of Claim 2. Consider $x_{0} \in \mathcal{J}_{p}\left(\omega_{1}\right)$ defined by

$$
x_{0}(\alpha)= \begin{cases}1, & \text { if } \sup \bigcup_{i \in \mathbb{N}} \Delta_{i}<\alpha \leqslant \sup \bigcup_{i \in \mathbb{N}} F_{\beta_{i}, k_{i}}, \\ 0, & \text { otherwise. }\end{cases}
$$

Plainly, $\rho_{k_{i}}\left(x_{0}\right)=0$ and $\psi_{\beta_{i}, k_{i}}\left(x_{0}\right)=\sum_{\left|\Delta_{k_{i}}\right|<j \leqslant m_{k_{i}}} q_{k_{i}, j}$ for every $i \in \mathbb{N}$, thus the convergence $\varphi_{\beta_{i}, k_{i}}\left(x_{0}\right) \rightarrow \varphi_{0}\left(x_{0}\right)$ implies the first part of the claim with $\lambda=\varphi_{0}\left(x_{0}\right)$. To complete the argument let

$$
X=\overline{\operatorname{span}}\left\{\mathbf{1}_{[0, \alpha]}: 0 \leqslant \alpha \leqslant \sup \bigcup_{i \in \mathbb{N}} \Delta_{i}\right\}
$$

and observe that for each $x \in X$ we have $\rho_{k_{i}}(x)=\varphi_{\beta_{i}, k_{i}}(x) \rightarrow \varphi_{0}(x)$. Obviously, $\mathcal{J}_{p}\left(\omega_{1}\right)=$ $X \oplus Y$, where $Y$ consists of all sequences $x \in \mathcal{J}_{p}\left(\omega_{1}\right)$ with $x(\alpha)=0$ for each $\alpha \leqslant \sup \bigcup_{i \in \mathbb{N}} \Delta_{i}$. For $y \in Y$ and every $i \in \mathbb{N}$ we have $\rho_{k_{i}}(y)=0$, hence our assertion holds true with $\rho$ defined by $\rho(x+y)=\varphi_{0}(x)$ for $x \in X$ and $y \in Y$.

Now, define $D \subset \omega_{1}$ to be the set of all ordinals $\alpha \in \omega_{1}$ for which there exists a sequence $\left(\alpha_{i}\right)_{i=1}^{\infty} \subset \omega_{1}$ satisfying:

(1) $\left(\alpha_{i}\right)_{i=1}^{\infty}$ is strictly increasing;

(2) $\alpha_{i} \in A_{k_{i}}$ for each $i \in \mathbb{N}$;

(3) $\lim _{i \rightarrow \infty} \alpha_{i}=\lim _{i \rightarrow \infty} \min \left(F_{\alpha_{i}, k_{i}} \backslash \Delta_{k_{i}}\right)=\alpha$;

(4) $\max \left(F_{\alpha_{i}, k_{i}}\right)<\alpha_{i+1}<\alpha$ for each $i \in \mathbb{N}$. 
It is clear that $D$ is then a club subset of $\omega_{1}$.

Claim 3. If $\left(\alpha_{i}\right)_{i=1}^{\infty} \subset \omega_{1}$ satisfies conditions (1)-(4) and converges to an $\alpha \in D$, then $\varphi_{\alpha_{i}, k_{i}} \underset{i \rightarrow \infty}{\stackrel{w *}{\longrightarrow}} \rho+\lambda e_{\alpha}^{*}$.

Proof of Claim 3. For every $x \in \mathcal{J}_{p}\left(\omega_{1}\right)$ we have

$$
\begin{aligned}
\left|\varphi_{\alpha_{i}, k_{i}}(x)-\left(\rho+\lambda e_{\alpha}^{*}\right)(x)\right| & \leqslant\left|\left(\rho_{k_{i}}-\rho\right)(x)\right|+\left|\psi_{\alpha_{i}, k_{i}}(x)-\lambda e_{\alpha}^{*}(x)\right| \\
& \leqslant\left|\left(\rho_{k_{i}}-\rho\right)(x)\right|+\left|\sum_{\left|\Delta_{k_{i}}\right|<j \leqslant m_{k_{i}}} q_{k_{i}, j} e_{\alpha}^{*}(x)-\lambda e_{\alpha}^{*}(x)\right| \\
& +\left|\sum_{\left|\Delta_{k_{i}}\right|<j \leqslant m_{k_{i}}} q_{k_{i}, j}\left(e_{\sigma_{\alpha_{i}, k_{i}}(j)}^{*}(x)-e_{\alpha}^{*}(x)\right)\right| .
\end{aligned}
$$

By Claim 2, the first two terms tend to zero as $i \rightarrow \infty$.

Let $X_{\alpha}$ be the set of all sequences $x \in \mathcal{J}_{p}\left(\omega_{1}\right)$ which are constant on some neighbourhood of $\alpha$. If $x \in X_{\alpha}$ then conditions (3) and (4) guarantee that for sufficiently large $i$ 's every summand in the last term equals zero. Consequently, $\varphi_{\alpha_{i}, k_{i}}(x) \rightarrow\left(\rho+\lambda e_{\alpha}^{*}\right)(x)$ is valid for every $x \in X_{\alpha}$, hence also for every $x \in \operatorname{span} X_{\alpha}$, so also for $x \in \overline{\operatorname{span}} X_{\alpha}$, since $\varphi_{\alpha_{i}, k_{i}}$ are equicontinuous. But $X_{\alpha}$ is linearly dense in $\mathcal{J}_{p}\left(\omega_{1}\right)$, as it contains each element of the Schauder basis.

To complete the proof let again $\alpha \in D$ be the limit of a sequence $\left(\alpha_{i}\right)_{i=1}^{\infty} \subset \omega_{1}$ satisfying (1)-(4). By Claim 1, we have $\varphi_{\alpha_{i}, k_{i}} \stackrel{w *}{\longrightarrow} \varphi_{\alpha}$, whence Claim 3 yields $\varphi_{\alpha}=\rho+\lambda e_{\alpha}^{*}$.

Since this is true for every $\alpha \in D$, we may pass to the limit with $\alpha \rightarrow \omega_{1}(\alpha \in D)$ to get $\varphi_{\alpha} \stackrel{w *}{\longrightarrow} \rho$ and, on the other hand, $\varphi_{\alpha}=T^{*} e_{\alpha}^{*} \stackrel{w *}{\longrightarrow} 0$. Therefore, $\rho=0$, thus $\varphi_{\alpha}=\lambda e_{\alpha}^{*}$ for each $\alpha \in D$.

Now, as in [17], we define a map $\Lambda_{p}: \mathscr{B}\left(\mathcal{J}_{p}\left(\omega_{1}\right)\right) \rightarrow \mathbb{K}$ by $\Lambda_{p}(T)=\lambda$, where $\lambda \in \mathbb{K}$ is chosen such that (3.1) holds (the uniqueness of such a $\lambda$, for fixed $T$, follows from the fact that the intersection of two club subsets of $\omega_{1}$ is again a club subset). Obviously, $\Lambda_{p}$ is a non-zero linear and multiplicative functional, hence $\operatorname{ker} \Lambda_{p}$ is a (maximal) ideal in $\mathscr{B}\left(\mathcal{J}_{p}\left(\omega_{1}\right)\right)$ of codimension one.

Let $L\left(\omega_{1}\right)$ be the set of all non-zero limit ordinals less than $\omega_{1}$. For every ordinal $\alpha \in\left(0, \omega_{1}\right)$ we define a subspace of $\mathcal{J}_{p}\left(\omega_{1}\right)$ by

$$
\mathcal{J}_{p}(\alpha)=\overline{\operatorname{span}}\left\{\mathbf{1}_{[0, \beta]}: 0 \leqslant \beta<\alpha\right\}
$$

and we let

$$
\mathfrak{G}_{p}=\left(\bigoplus_{\alpha \in L\left(\omega_{1}\right)} \mathcal{J}_{p}(\alpha)\right)_{\ell_{p}} .
$$

Being an $\ell_{p}$-sum of WCG (even separable) Banach spaces, with $p \in(1, \infty)$, the space $\mathfrak{G}_{p}$ is a WCG Banach space (cf. [26, Proposition 2.4]). It turns out that weakly compactly generated operators on the long James space factor through this concrete WCG space, just like weakly compact operators on the classical James space factor through the concrete reflexive space $\left(\bigoplus_{n=1}^{\infty} \mathcal{J}_{p}(n)\right)_{\ell_{p}}$ identified by Laustsen. 
The estimates given by Casazza, Lin and Lohman in [5, Lemma 2] for the classical James space and $p=2$ can be easily generalised in the following manner.

Lemma 3.2. Let $p \in(1, \infty)$ and $k, n_{1}, \ldots, n_{k} \in \mathbb{N}$. For any ordinal numbers

$$
\gamma_{1,1}<\ldots<\gamma_{1, n_{1}}<\ldots<\gamma_{k, 1}<\ldots<\gamma_{k, n_{k}}<\omega_{1}
$$

satisfying $\gamma_{i, n_{i}}+1<\gamma_{i+1,1}$ for each $1 \leqslant i<k$, and for any scalars $t_{i, j}$, we have

$$
\sum_{i=1}^{k}\left\|\sum_{j=1}^{n_{i}} t_{i, j} e_{\gamma_{i, j}}\right\|_{p, 0}^{p} \leqslant\left\|\sum_{i=1}^{k} \sum_{j=1}^{n_{i}} t_{i, j} e_{\gamma_{i, j}}\right\|_{p, 0}^{p} \leqslant 2^{p-1} \sum_{i=1}^{k}\left\|\sum_{j=1}^{n_{i}} t_{i, j} e_{\gamma_{i, j}}\right\|_{p, 0}^{p} .
$$

Proof. The first inequality is obvious, since for each $1 \leqslant i \leqslant k$ the $i$ th summand on the lefthand side may be calculated using only indices from the interval $\left(\gamma_{i-1, n_{i-1}}, \gamma_{i+1,1}\right)$, where we put $\gamma_{0, n_{0}}=-1$ and $\gamma_{k+1,1}=\omega_{1}$.

For the second estimate notice that convexity of the function $x \mapsto|x|^{p}$ gives $|t+u|^{p} \leqslant$ $2^{p-1}\left(|t|^{p}+|u|^{p}\right)$ for $t, u \in \mathbb{C}$. Consequently, for $t, u \in \mathbb{C}$ we have

$$
|t-u|^{p} \leqslant 2^{p-1}\left(|t-0|^{p}+|0-u|^{p}\right)
$$

thus we can change every estimate under the supremum sign defining the middle term into a sum which does not exceed the right-hand side.

Proposition 3.3. For each $p \in(1, \infty)$ the space $\mathcal{J}_{p}\left(\omega_{1}\right)$ contains a complemented copy of $\mathfrak{G}_{p}$ with $\mathcal{J}_{p}\left(\omega_{1}\right) \simeq \mathfrak{G}_{p} \oplus \mathcal{J}_{p}\left(\omega_{1}\right)$.

Proof. For $\alpha<\omega_{1}$ let us define a subspace of $\widetilde{\mathcal{J}}_{p}$ by $\widetilde{\mathcal{J}}_{p}(\alpha)=\overline{\operatorname{span}}\left\{e_{\beta}: 0 \leqslant \beta<\alpha\right\}$. The order preserving bijection $\varphi: \omega_{1} \rightarrow D\left(\omega_{1}\right) \cup\{0\}$ induces a surjective isometry between $\widetilde{\mathcal{J}}_{p}(\alpha)$ and $\mathcal{J}_{p}(\alpha)$ for each $\alpha \in L\left(\omega_{1}\right)$. Hence, $\mathfrak{G}_{p}$ is isometric to $\widetilde{\mathfrak{G}}_{p}$, where

$$
\widetilde{\mathfrak{G}}_{p}=\left(\bigoplus_{\alpha \in L\left(\omega_{1}\right)} \widetilde{\mathcal{J}}_{p}(\alpha)\right)_{\ell_{p}}
$$

It is enough to prove that the space $\widetilde{\mathcal{J}}_{p}\left(\omega_{1}\right)$ contains a complemented copy of $\widetilde{\mathfrak{G}}_{p}$.

CLAIM 1. The canonical basis of $\widetilde{\mathfrak{G}}_{p}$ is equivalent to a basic sequence $\left(e_{\alpha}\right)_{\alpha \in A} \subset \widetilde{\mathcal{J}}_{p}\left(\omega_{1}\right)$ with a certain set $A \subset \omega_{1}$.

Proof of Claim 1. Define

$$
\Gamma=\left\{(\alpha, \beta) \in \omega_{1} \times \omega_{1}: \alpha \in L\left(\omega_{1}\right) \text { and } 0 \leqslant \beta<\alpha\right\}
$$

and let $\Gamma^{*}=\Gamma \cup \omega_{1}$. Consider a linear order $\prec$ on $\Gamma^{*}$ defined as follows:

(i) $\left.\prec\right|_{\Gamma}$ is the lexicographic order;

(ii) $\left.\prec\right|_{\omega_{1}}$ is the natural order;

(iii) $(\beta, \gamma)<\alpha<(\delta, \varepsilon)$ for every $(\beta, \gamma),(\delta, \varepsilon) \in \Gamma$ with $\beta \leqslant \alpha<\delta$.

By a standard recursive argument, we infer that $\left(\Gamma^{*}, \prec\right)$ is order-isomorphic to $\omega_{1}$. Let $\psi: \Gamma^{*} \rightarrow \omega_{1}$ be the order-isomorphism.

Now, suppose $k, n_{1}, \ldots, n_{k} \in \mathbb{N}$ and we are given ordinal numbers $\alpha_{1}<\ldots<\alpha_{k} \in L\left(\omega_{1}\right)$ and $\beta_{i, 1}<\ldots<\beta_{i, n_{i}}<\alpha_{i}$ (for $1 \leqslant i \leqslant k$ ) such that $\left(\alpha_{i}, \beta_{i, j}\right) \in \Gamma$. Let $x$ be the element 
of $\widetilde{\mathfrak{G}}_{p}$ whose $\alpha_{i}$ th coordinate equals $\sum_{j=1}^{n_{i}} t_{i, j} e_{\beta_{i, j}}$ for $1 \leqslant i \leqslant k$ (with some scalars $t_{i, j}$ ), and whose all other coordinates are zeros. Let also $\gamma_{i, j}=\psi\left(\alpha_{i}, \beta_{i, j}\right)$ for $1 \leqslant i \leqslant k$ and $1 \leqslant j \leqslant n_{i}$.

Define $y \in \widetilde{\mathcal{J}}_{p}\left(\omega_{1}\right)$ by the formula $y=\sum_{i=1}^{k} \sum_{j=1}^{n_{i}} t_{i, j} e_{\gamma_{i, j}}$. Since

$$
\gamma_{i, n_{i}}=\psi\left(\alpha_{i}, \beta_{i, n_{i}}\right)<\psi\left(\alpha_{i}\right)<\psi\left(\alpha_{i+1}, \beta_{1}\right)=\gamma_{i+1,1} \quad \text { for } 1 \leqslant i<k,
$$

an application of Lemma 3.2 yields

$$
\|x\|_{\widetilde{\mathfrak{G}}_{p}}^{p} \leqslant\|y\|_{p, 0}^{p} \leqslant 2^{p-1}\|x\|_{\widetilde{\mathfrak{G}}_{p}}^{p} .
$$

Thus there is an isomorphism witnessing that the canonical basis of $\widetilde{\mathfrak{G}}_{p}$ is equivalent to $\left(e_{\alpha}\right)_{\alpha \in A}$ with $A=\psi(\Gamma) \subset \omega_{1}$.

The next claim will complete the proof.

Claim 2. The subspace $X=\overline{\operatorname{span}}\left\{e_{\alpha}: \alpha \in A\right\}$ is complemented in $\widetilde{\mathcal{J}}_{p}\left(\omega_{1}\right)$ by a copy of $\widetilde{\mathcal{J}}_{p}\left(\omega_{1}\right)$.

Let $B=\left(\omega_{1} \backslash A\right) \cup\{-1\}$. Following the lines of the proof of [5, Theorem 5], consider two sets:

$$
\begin{array}{r}
C=\left\{\mathbf{1}_{[\alpha, \beta]} \in \widetilde{\mathcal{J}}_{p}\left(\omega_{1}\right): \alpha \in A, \text { either } \alpha \in L\left(\omega_{1}\right) \text { or } \alpha=\alpha^{\prime}+1 \text { with } \alpha^{\prime} \in B,\right. \\
\text { whereas } \left.\beta=\min \left\{\beta^{\prime} \in B: \beta^{\prime}>\alpha\right\}\right\}
\end{array}
$$

and

$$
D=\left\{e_{\beta} \in \widetilde{\mathcal{J}}_{p}\left(\omega_{1}\right): \beta \in B \text {, either } \beta \in L\left(\omega_{1}\right) \text { or } \beta=\beta^{\prime}+1 \text { with } \beta^{\prime} \in B\right\},
$$

and define $Y=\overline{\operatorname{span}}(C \cup D)$. Then for $x \in X$ and $y \in Y$ we have $\|y\|_{p, 0} \leqslant\|x+y\|_{p, 0}$, since every partial variation approximating $\|y\|_{p, 0}$ may be calculated for coordinates from $B$, and it remains the same for $x+y$. Therefore, $\|x\|_{p, 0} \leqslant 2\|x+y\|_{p, 0}$, thus there is a projection $P$ on $\widetilde{\mathcal{J}}_{p}\left(\omega_{1}\right)$, with range $X$ and kernel $Y$, and $\|P\| \leqslant 2$.

The elements of $C \cup D$ form a block basic sequence of $\left(e_{\alpha}\right)_{\alpha<\omega_{1}}$ which is plainly equivalent to $\left(e_{\alpha}\right)_{\alpha<\omega_{1}}$. Thus $Y \simeq \widetilde{\mathcal{J}}_{p}\left(\omega_{1}\right)$ and the proof is completed.

Lemma 3.4. For each $p \in(1, \infty)$ we have $\mathfrak{G}_{p} \simeq \ell_{p}\left(\omega_{1}, \mathfrak{G}_{p}\right)$ (the $\ell_{p}$-sum of $\omega_{1}$ copies of $\mathfrak{G}_{p}$ ). Consequently, by Petczyński decomposition method, $\mathfrak{G}_{p}$ is also isomorphic to the $\ell_{p}$-sum of countably many copies of itself.

Proof. By using transfinite induction, with respect to the lexicographic order on the set $\omega_{1} \times L\left(\omega_{1}\right)$, we construct a one-to-one map $\theta: \omega_{1} \times L\left(\omega_{1}\right) \rightarrow L\left(\omega_{1}\right)$ such that $\theta(\alpha, \beta)>\beta$ for every $(\alpha, \beta) \in \omega_{1} \times L\left(\omega_{1}\right)$.

For $(\alpha, \beta) \in \omega_{1} \times L\left(\omega_{1}\right)$ let $\iota_{\alpha, \beta}: \mathcal{J}_{p}(\beta) \rightarrow \mathcal{J}_{p}(\theta(\alpha, \beta))$ be the embedding of the $\beta$ th summand of the $\alpha$ th coordinate of $\ell_{p}\left(\omega_{1}, \mathfrak{G}_{p}\right)$ into $\mathcal{J}_{p}(\theta(\alpha, \beta))$ which just puts the sequences from $\mathcal{J}_{p}(\beta)$ into the first $\beta$ coordinates. Let $\iota: \ell_{p}\left(\omega_{1}, \mathfrak{G}_{p}\right) \rightarrow \mathfrak{G}_{p}$ be the embedding naturally produced by all $\iota_{\alpha, \beta}$ 's.

Obviously, $\iota$ is an isometric embedding, thus $\iota\left(\ell_{p}\left(\omega_{1}, \mathfrak{G}_{p}\right)\right)$ is a closed subspace of $\mathfrak{G}_{p}$. Since every summand $\mathcal{J}_{p}(\theta(\alpha, \beta))$ of $\mathfrak{G}_{p}$ admits a natural, norm one projection onto $\mathcal{J}_{p}(\beta)$, there also exists a norm one projection from $\mathfrak{G}_{p}$ onto $\iota\left(\ell_{p}\left(\omega_{1}, \mathfrak{G}_{p}\right)\right)$. Thus, $\ell_{p}\left(\omega_{1}, \mathfrak{G}_{p}\right)$ is 
isomorphic to a complemented subspace of $\mathfrak{G}_{p}$ and, obviously, vice versa. Moreover, both spaces $\mathfrak{G}_{p}$ and $\ell_{p}\left(\omega_{1}, \mathfrak{G}_{p}\right)$ are clearly isomorphic to their squares. Hence, by the Pełczyński decomposition method, we get the assertion.

The following two assertions can be proved in the same manner as Proposition 2.1 and Corollary 2.3 in [17], so we omit their proofs.

Lemma 3.5. If $T \in \mathscr{B}\left(\mathcal{J}_{p}\left(\omega_{1}\right)\right)$ and $\Lambda_{p}(T) \neq 0$ then $T$ fixes a complemented copy of $\mathcal{J}_{p}\left(\omega_{1}\right)$ and the range of $T$ contains a copy of $\mathcal{J}_{p}\left(\omega_{1}\right)$, complemented in $\mathcal{J}_{p}\left(\omega_{1}\right)$.

Corollary 3.6. If $Y \subset \mathcal{J}_{p}\left(\omega_{1}\right)$ and $Y \simeq \mathcal{J}_{p}\left(\omega_{1}\right)$ then there is a subspace $Z \subseteq Y$ which is complemented in $\mathcal{J}_{p}\left(\omega_{1}\right)$ and such that $Z \simeq \mathcal{J}_{p}\left(\omega_{1}\right)$.

Before we proceed to the main result of this section, we require another piece of notation.

Following Dosev and Johnson [11], for each Banach space $X$ we define

$$
\mathscr{M}_{X}=\{T \in \mathscr{B}(X): I \neq A T B(A, B \in \mathscr{B}(X))\} .
$$

In general, the set $\mathscr{M}_{X}$ need not be closed under addition but when it is, it is also the unique maximal ideal of $\mathscr{B}(X)$. A recent result of the first-named author and Laustsen [18, Theorem 1.2] states that $\mathscr{M}_{C\left[0, \omega_{1}\right]}$ is the unique maximal ideal of $\mathscr{B}\left(C\left[0, \omega_{1}\right]\right)$.

Now, we are ready to prove the following theorem.

Theorem 3.7. For any $p \in(1, \infty)$ we have

$$
\operatorname{ker} \Lambda_{p}=\mathscr{W} \mathscr{C} \mathscr{G}\left(\mathcal{J}_{p}\left(\omega_{1}\right)\right)=\mathscr{G}_{\mathfrak{G}_{p}}\left(\mathcal{J}_{p}\left(\omega_{1}\right)\right)=\mathscr{C}\left(\mathcal{J}_{p}\left(\omega_{1}\right)\right)=\mathscr{M}_{\mathcal{J}_{p}\left(\omega_{1}\right)},
$$

and this is the unique maximal ideal of $\mathscr{B}\left(\mathcal{J}_{p}\left(\omega_{1}\right)\right)$. Moreover, an operator $T \in \mathscr{B}\left(\mathcal{J}_{p}\left(\omega_{1}\right)\right)$ belongs to this ideal if and only if it is $\mathcal{J}_{p}\left(\omega_{1}\right)$-singular, that is, $T$ does not fix a copy of $\mathcal{J}_{p}\left(\omega_{1}\right)$.

Proof. Let us start by showing that the ideal ker $\Lambda_{p}$ is contained in each of the remaining sets in the desired equality. So, suppose $T \in \mathscr{B}\left(\mathcal{J}_{p}\left(\omega_{1}\right)\right)$ and $\left\langle e_{\alpha}^{*}, T x\right\rangle=0$ for every $x \in \mathcal{J}_{p}\left(\omega_{1}\right)$ and $\alpha \in D$, where $D$ is a certain club subset of $\omega_{1}$. We may assume that $D \subseteq L\left(\omega_{1}\right)$.

Define $Y$ to be the set of all sequences $x \in \mathcal{J}_{p}\left(\omega_{1}\right)$ such that $x(\alpha)=0$ for every $\alpha \in D$. Obviously, the range of $T$ lies in $Y$. By Lemma [3.2, the space $Y$ is in turn isomorphic to $\left(\bigoplus_{\alpha<\omega_{1}} \widetilde{\mathcal{J}}_{p}\left(\operatorname{ord} O_{\alpha}\right)\right)_{\ell_{p}}$, where $\left\{O_{\alpha}: \alpha<\omega_{1}\right\}$ is the family of consecutive order-components of $\omega_{1} \backslash D$ (this family is uncountable as $\left.D \subset L\left(\omega_{1}\right)\right)$ and ord $O_{\alpha}$ denotes the order type of $O_{\alpha}\left(\alpha<\omega_{1}\right)$. That space is isomorphic to a complemented subspace of $\widetilde{\mathfrak{G}}_{p}$ given by (??). Hence, by Proposition 3.3, it is isomorphic to a subspace of $\mathcal{J}_{p}\left(\omega_{1}\right)$ and is, of course, weakly compactly generated. Thus, the inclusion ker $\Lambda_{p} \subset \mathscr{W} \mathscr{C} \mathscr{G}\left(\mathcal{J}_{p}\left(\omega_{1}\right)\right)$ has been proved. Furthermore, $\Lambda_{p}(T)=0$ implies $T \in \mathscr{G}_{\mathfrak{G}_{p}}\left(\mathcal{J}_{p}\left(\omega_{1}\right)\right)$, as in this case the range of $T$ is contained in a subspace of $\mathfrak{G}_{p}$.

Now, observe that Proposition 3.3 yields

$$
\mathcal{J}_{p}\left(\omega_{1}\right) \cong \mathfrak{G}_{p} \oplus \mathcal{J}_{p}\left(\omega_{1}\right) \cong \mathfrak{G}_{p} \oplus \mathfrak{G}_{p} \oplus \mathcal{J}_{p}\left(\omega_{1}\right) \cong \ldots,
$$

thus every operator factoring through $\mathfrak{G}_{p}$ is compressible. Consequently, $\operatorname{ker} \Lambda_{p} \subset \mathscr{C}\left(\mathcal{J}_{p}\left(\omega_{1}\right)\right)$. 
To show the last of the announced inclusions recall that $\operatorname{ker} \Lambda_{p} \subset \mathscr{W} \mathscr{C} \mathscr{G}\left(J_{p}\left(\omega_{1}\right)\right)$ and observe that the identity of $\mathcal{J}_{p}\left(\omega_{1}\right)$ cannot factor through a weakly compactly generated operator, as $J_{p}\left(\omega_{1}\right)$ is not weakly compactly generated. Hence, $\operatorname{ker} \Lambda_{p} \subseteq \mathscr{M}_{\mathcal{J}_{p}\left(\omega_{1}\right)}$.

Since ker $\Lambda_{p}$ is a maximal ideal, and the ideals $\mathscr{W} \mathscr{C} \mathscr{G}\left(\mathcal{J}_{p}\left(\omega_{1}\right)\right)$ and $\mathscr{G}_{\mathfrak{G}_{p}}\left(\mathcal{J}_{p}\left(\omega_{1}\right)\right)$ are clearly proper, we get the first two of the claimed equalities.

To show the equality $\operatorname{ker} \Lambda_{p}=\mathscr{C}\left(\mathcal{J}_{p}\left(\omega_{1}\right)\right)$ we shall prove that the identity operator $I_{\mathcal{J}_{p}\left(\omega_{1}\right)}$ is not compressible.

By [31, Proposition 2], that would be the case if and only if for a certain $n \in \mathbb{N}$ we had a decomposition

$$
\mathcal{J}_{p}\left(\omega_{1}\right)^{n} \cong Z \oplus \mathcal{J}_{p}\left(\omega_{1}\right)^{n+1}
$$

with some Banach space $Z$. However, as the ideal $\mathscr{W} \mathscr{C} \mathscr{G}\left(\mathcal{J}_{p}\left(\omega_{1}\right)\right)$ has codimension one in $\mathscr{B}\left(\mathcal{J}_{p}\left(\omega_{1}\right)\right)$ hence there is no such a decomposition.

For the equality $\operatorname{ker} \Lambda_{p}=\mathscr{M}_{\mathcal{J}_{p}\left(\omega_{1}\right)}$ it remains to prove the inclusion " $\supset$ ", but this will follow from the fact that any operator $T$ with $\Lambda_{p}(T) \neq 0$ must fix a copy of $\mathcal{J}_{p}\left(\omega_{1}\right)$ and, consequently, the identity factors through $T$. These two statements are contained in the remaining part of the proof.

Now, notice that since $\Lambda_{p}(T)=0$ implies $T \in \mathscr{W} \mathscr{C} \mathscr{G}\left(\mathcal{J}_{p}\left(\omega_{1}\right)\right)$, it implies also that $T$ does not fix a copy of $\mathcal{J}_{p}\left(\omega_{1}\right)$. The converse follows from Lemma 3.5. Hence, each of the ideals listed in our assertion is just the set of operators not fixing a copy of $\mathcal{J}_{p}\left(\omega_{1}\right)$. We conclude here that $\operatorname{ker} \Lambda_{p}=\mathscr{M}_{\mathcal{J}_{p}\left(\omega_{1}\right)}$ is the unique maximal ideal of $\mathscr{B}\left(J_{p}\left(\omega_{1}\right)\right)$.

Corollary 3.8. The range of a weakly compactly generated operator on $\mathcal{J}_{p}\left(\omega_{1}\right)$ is contained in a complemented, WCG subspace of $\mathcal{J}_{p}\left(\omega_{1}\right)$.

Let us say that a sequence $\left(A_{\xi}\right)_{\xi<\omega_{1}}$ of subsets of $\omega_{1}$ is skipped if for each pair $\xi_{1}<\xi_{2}<\omega_{1}$ we have $\sup A_{\xi_{1}}+1<\min A_{\xi_{2}}$. A sequence $\left(f_{\xi}\right)_{\xi<\omega_{1}}$ of functions defined on $\omega_{1}$ is skipped provided the sequence $\left(\operatorname{supp} f_{\xi}\right)_{\xi<\omega_{1}}$ is skipped.

The next lemma is an easy consequence of Lemma 3.2 .

Lemma 3.9. For each $p \in(1, \infty)$ any normalised skipped sequence $\left(f_{\xi}\right)_{\xi<\omega_{1}}$ in $\mathcal{J}_{p}\left(\omega_{1}\right)$ is equivalent to the canonical basis of $\ell_{p}\left(\omega_{1}\right)$.

For each $\sigma<\omega_{1}$ let us define $P_{\sigma} f=f \cdot \mathbf{1}_{[0, \sigma]}, f \in \mathcal{J}_{p}\left(\omega_{1}\right)$. We note that $P_{\sigma}$ is a welldefined contractive projection on $\mathcal{J}_{p}\left(\omega_{1}\right)$. The proof of the next theorem is based on ideas from the proofs of [18, Theorem 1.3, Lemma 4.3].

Theorem 3.10. Let $p \in(1, \infty)$. The following assertions are equivalent for an operator $T$ on $\mathcal{J}_{p}\left(\omega_{1}\right)$ :

(a) $T=T P_{\sigma}$ for some countable ordinal $\sigma$;

(b) $T \in \mathscr{G}_{\mathcal{J}_{p}(\sigma)}\left(\mathcal{J}_{p}\left(\omega_{1}\right)\right)$ for some countable ordinal $\sigma$;

(c) $T \in \overline{\mathscr{G}}_{\mathcal{J}_{p}(\sigma)}\left(\mathcal{J}_{p}\left(\omega_{1}\right)\right)$ for some countable ordinal $\sigma$;

(d) $T \in \mathscr{X}\left(\mathcal{J}_{p}\left(\omega_{1}\right)\right)$;

(e) $T$ does not fix a copy of $\ell_{p}\left(\omega_{1}\right)$.

Proof. The implications (国) $\Rightarrow(\underline{b}) \Rightarrow(\underline{\mathrm{c}}) \Rightarrow(\underline{\mathrm{d}}) \Rightarrow($ 远 $)$ are clear. 
Assume contrapositively that (a) fails. We claim that there is $\delta>0$ such that for each $\xi<\omega_{1}$, there is $f_{\xi} \in \mathcal{J}_{p}\left(\omega_{1}\right)$ with $\operatorname{supp} f_{\xi} \subseteq\left(\xi, \omega_{1}\right),\|T f\| \geqslant \delta$ and $\|f\| \leqslant 1$. Suppose this is not the case. Then, for $\delta_{n}=1 / n$ we obtain a sequence $\left(\xi_{n}\right)_{n \in \mathbb{N}}$ of countable ordinals such that $\|T f\|<1 / n$ for each $f \in \mathcal{J}_{p}\left(\omega_{1}\right)$ with $\operatorname{supp} f \subseteq\left(\xi_{n}, \omega_{1}\right)$. Let $\xi=\sup \left\{\xi_{n}: n \in \mathbb{N}\right\}$. Certainly, $\xi<\omega_{1}$. Take $g \in \mathcal{J}_{p}\left(\omega_{1}\right)$ with $\left\|\left(I-P_{\xi}\right) g\right\| \leqslant 1$. Letting $f=\left(I-P_{\xi}\right) g$ we infer that $\operatorname{supp} f \subseteq\left(\xi, \omega_{1}\right)=\bigcap_{n \in \mathbb{N}}\left(\xi_{n}, \omega_{1}\right)$ as $P_{\xi}\left(I-P_{\xi}\right)=0$. Thus, $\|T f\|<1 / n$ for each $n \in \mathbb{N}$, so $0=T f=T\left(I-P_{\xi}\right) g$, which proves $T=T P_{\xi}$, against the assumption.

Similarly, as in the proof [18, Theorem 1.3], we choose inductively a normalised skipped sequence $\left(f_{\xi}\right)_{\xi<\omega_{1}}$ with $\left\|T f_{\xi}\right\| \geqslant \delta$, where $\delta$ is as above. By Lemma 3.9, the subspace $X=\overline{\operatorname{span}}\left\{f_{\xi}: \xi<\omega_{1}\right\}$ is isomorphic to $\ell_{p}\left(\omega_{1}\right)$. We note that $\left.T\right|_{X}$ is bounded below, hence the proof of the implication (国) $\Rightarrow$ (国) is complete.

An element $x$ of an algebra $\mathscr{A}$ is a commutator if there exist some $a, b \in \mathscr{A}$ such that $x=a b-b a$. It is well-known that if $\mathscr{A}$ is a unital Banach algebra, then its unit cannot be a commutator. The following result is the counterpart for $\mathcal{J}_{p}\left(\omega_{1}\right)$ of [22, Theorem 4.6] and [17, Theorem 5.1] for $C\left[0, \omega_{1}\right]$.

Theorem 3.11. Each operator in $\mathscr{W} \mathscr{C} \mathscr{G}\left(\mathcal{J}_{p}\left(\omega_{1}\right)\right)$ is a sum of three commutators.

Proof. We have that $\mathscr{W} \mathscr{C} \mathscr{G}\left(\mathcal{J}_{p}\left(\omega_{1}\right)\right)=\mathscr{G}_{\mathfrak{G}_{p}}\left(\mathcal{J}_{p}\left(\omega_{1}\right)\right)$ and, by Lemma 3.4 , the space $\mathfrak{G}_{p}$ is isomorphic to its $\ell_{p}$-sum, so [22, Proposition 3.7] yields that each operator on $\mathfrak{G}_{p}$ is a sum of two commutators. Consequently, it follows from [22, Lemma 4.5] that each operator which factors through $\mathfrak{G}_{p}$ is a sum of three commutators.

Let $\vartheta: \mathscr{A} \rightarrow \mathscr{C}$ be a homomorphism between Banach algebras. We call

$$
\mathcal{I}:=\{a \in \mathscr{A} \text { : the maps } b \mapsto \vartheta(a b), b \mapsto \vartheta(b a) \text { are continuous }\}
$$

the continuity ideal of $\vartheta$. Certainly, $\mathcal{I}$ is a two-sided ideal of $\mathscr{A}$. Suppose $\mathscr{A}$ is an ideal of $\mathscr{B}(E)$, where $E$ is some Banach space. Willis [31, Proposition 7] proved that $\mathscr{A} \cdot \mathscr{C}(E) \cdot \mathscr{A} \subseteq$ $\mathcal{I}$ (recall that $\mathscr{C}(E)$ stands for the ideal of compressible operators). Furthermore, he used this fact to prove that each homomorphism from $\mathscr{B}\left(\mathcal{J}_{2}\right)$ is continuous (in fact, his argument extends to arbitrary $p \in(1, \infty))$. A key-ingredient in Willis' proof is existence of a bounded right approximate identity in the ideal of weakly compact operators on the James space; we shall prove that every homomorphism from $\mathscr{B}\left(\mathcal{J}_{p}\left(\omega_{1}\right)\right)$ is continuous without appealing to those type of results.

Theorem 3.12. Every homomorphism from $\mathscr{B}\left(\mathcal{J}_{p}\left(\omega_{1}\right)\right)$ is automatically continuous.

Proof. Let $\mathscr{C}$ be a Banach algebra and let $\vartheta: \mathscr{B}\left(\mathcal{J}_{p}\left(\omega_{1}\right)\right) \rightarrow \mathscr{C}$ be a homomorphism. As $\mathscr{W} \mathscr{C} \mathscr{G}\left(\mathcal{J}_{p}\left(\omega_{1}\right)\right)$ is equal to the ideal of compressible operators on $\mathcal{J}_{p}\left(\omega_{1}\right)$, the aforementioned result of Willis yields that $\mathscr{W} \mathscr{C} \mathscr{G}\left(\mathcal{J}_{p}\left(\omega_{1}\right)\right)$ is contained in the continuity ideal $\mathcal{I}$ of $\vartheta$.

Since the ideal of weakly compactly generated operators on $\mathcal{J}_{p}\left(\omega_{1}\right)$ is of codimension one in $\mathscr{B}\left(\mathcal{J}_{p}\left(\omega_{1}\right)\right)$, it is sufficient to prove that $\vartheta$ restricted to $\mathscr{W} \mathscr{C} \mathscr{G}\left(\mathcal{J}_{p}\left(\omega_{1}\right)\right)$ is continuous.

Let $\left(T_{n}\right)_{n=1}$ be a sequence of WCG operators on $\mathcal{J}_{p}\left(\omega_{1}\right)$. We shall exhibit a weakly compactly generated operator $P$ such that $T_{n}=P T_{n}(n \in \mathbb{N})$, which by definition of the continuity ideal, would complete the proof. 
For each $n \in \mathbb{N}$ there is a weakly compactly generated subspace $X_{n}$ of $\mathcal{J}_{p}\left(\omega_{1}\right)$, containing the image of $T_{n}$ and which is, by Corollary 3.8, isomorphic to $\mathfrak{G}_{p}$. Let $X=\left(\bigoplus_{n \in \mathbb{N}} X_{n}\right)_{\ell_{p}}$. By Lemma 3.4, $X$ isomorphic to $\mathfrak{G}_{p}$, so it is also WCG. Define $J: X \rightarrow \mathcal{J}_{p}\left(\omega_{1}\right)$ by $J\left(x_{n}\right)_{n=1}^{\infty}=\sum_{n=1}^{\infty} x_{n} / n^{p}$. Let $U: Y \rightarrow X$ be an isomorphism, where $Y$ is a complemented subspace of $\mathcal{J}_{p}\left(\omega_{1}\right)$, isomorphic to $\mathfrak{G}_{p}$. Let $V=J U, V: Y \rightarrow \mathcal{J}_{p}\left(\omega_{1}\right)$. Because $Y$ is complemented in $\mathcal{J}_{p}\left(\omega_{1}\right)$, we may extend $V$ to $\mathcal{J}_{p}\left(\omega_{1}\right)$; let $V$ still stand for any fixed such an extension. Plainly, $V \in \mathscr{W} \mathscr{C} \mathscr{G}\left(\mathcal{J}_{p}\left(\omega_{1}\right)\right)$, so $\Lambda_{p}(V)=0$. Thus, there is a complemented copy $Z$ of $\mathfrak{G}_{p}$ containing the range of $V$. Let $P$ be a projection onto $Z$. It remains to notice that

$$
\bigcup_{n \in \mathbb{N}} \operatorname{im} T_{n} \subseteq \bigcup_{n \in \mathbb{N}} X_{n} \subseteq \operatorname{im} T=\operatorname{im} V \subseteq Z
$$

In particular, $T_{k}=P T_{k}$ for each $k \in \mathbb{N}$.

Now, consider the complex version of $\mathcal{J}_{p}\left(\omega_{1}\right)$ only. Recall that a linear (not necessarily bounded) functional $\tau$ on a complex algebra $\mathscr{A}$ is a trace if $\tau(a b)=\tau(b a)(a, b \in \mathscr{A})$. It follows from Theorem 3.11 that a linear functional $\tau$ on $\mathscr{B}\left(\mathcal{J}_{p}\left(\omega_{1}\right)\right)$ is a trace if and only if it satisfies the equation $\tau=\tau(I) \Lambda_{p}$. Thus, $\Lambda_{p}$ is the only normalised trace on $\mathscr{B}\left(\mathcal{J}_{p}\left(\omega_{1}\right)\right)$. Laustsen proved that the $K_{0}$-group of $\mathscr{B}\left(\mathcal{J}_{p}\right)$ is isomorphic to the additive group of integers [20, Theorem 4.6]; for the definition of the $K_{0}$-group consult e.g. 44. Laustsen's proof relies on the fact that $\mathscr{B}\left(\mathcal{J}_{p}\right)$ has a unique normalised trace with kernel being an ideal of operators factoring through a Banach space isomorphic to its $\ell_{p}$-sum. It turns out that his argument carries over to $\mathscr{B}\left(\mathcal{J}_{p}\left(\omega_{1}\right)\right)$, thus we obtain as a by-product the following fact:

Proposition 3.13. $K_{0}\left(\mathscr{B}\left(\mathcal{J}_{p}\left(\omega_{1}\right)\right) \cong \mathbb{Z}\right.$.

\section{WeAKLy COMPACTLY GENERATED OPERATORS ON $C(K)$-SPACES}

The aim of this section is to give some natural conditions which would guarantee that a given operator on a $C(K)$ space is weakly compactly generated. It is well-known that every such operator $T: C(K) \rightarrow X$ has a Riesz-type representation (cf. [10, Chapter 6]). Namely, there exists a weak*-countably additive vector measure $\mu: \Sigma \rightarrow X^{* *}$ (called the representing measure for $T$ ), defined on the $\sigma$-algebra $\Sigma$ of all Borel subsets of $K$, such that:

(i) for each $x^{*} \in X^{*}$ the map $\Sigma \ni A \rightarrow\left\langle\mu(A), x^{*}\right\rangle$ is a regular countably additive scalar measure (and will be denoted by $x^{*} \circ \mu$ );

(ii) $\left\langle x^{*}, T f\right\rangle=\int_{K} f \mathrm{~d}\left(x^{*} \circ \mu\right)$ for each $x^{*} \in X^{*}$ and $f \in C(K)$;

(iii) $\|T\|=\|\mu\|(K)$.

The representing measure $\mu$ of $T$ may be defined explicitly by

$$
\mu(A)=T^{* *} \mathbf{1}_{A} \quad(A \in \Sigma) .
$$

Equivalently,

$$
\left\langle\mu(A), x^{*}\right\rangle=\mu_{x^{*}}(A),
$$


where $\mu_{x^{*}}$ is the scalar measure produced by the Riesz theorem applied for the functional $x^{*} T$.

Suppose $K$ is an Eberlein compact space, that is, $K$ is homeomorphic to a weakly compact subset of some Banach space (we refer to [26] for an exposition concerning the class of Eberlein compact spaces), and consider the identity operator $I_{C(K)}$, which is weakly compactly generated. Then for every $A \in \Sigma$ we have $\mu(A)=\varphi_{A}$ and a straightforward calculation gives $\|\mu\|(A)=1$, provided that $A \neq \varnothing$. So, for a WCG operator on $C(K)$ it may happen that there are no non-empty sets of small semivariation, nonetheless in our example the whole domain is Eberlein. In this spirit we will prove the following result.

Theorem 4.1. Let $K$ be a compact Hausdorff space, $X$ be a Banach space, and $T: C(K) \rightarrow$ $X$ be a bounded operator. Suppose $\mu: \Sigma \rightarrow X^{* *}$ is the representing measure for $T$ and for each $\varepsilon>0$ there exists a decomposition $K=K_{E}^{\varepsilon} \cup L^{\varepsilon}$, where $K_{E}^{\varepsilon}$ is an Eberlein compactum and $\|\mu\|\left(L^{\varepsilon}\right)<\varepsilon$. Then the range of $T$ lies in a WCG Banach space.

We shall make use of the characterisation of subspaces of WCG Banach spaces, obtained by Fabian, Montesinos and Zizler [13] (cf. also [15, Theorem 6.13]). Recall that a subset $M$ of a Banach space $X$ is called $\varepsilon$-weakly compact if it is bounded and $\bar{M}^{w *} \subseteq X+\varepsilon B_{X^{* *}}$.

Theorem 4.2. A Banach space is a subspace of a WCG Banach space if and only if for every $\varepsilon>0$ its unit ball can be covered by countably many $\varepsilon$-weakly compact sets.

Proof of Theorem 4.1. Fix $\varepsilon>0$ and define $\hat{T}: C\left(K_{E}^{\varepsilon}\right) \rightarrow X$ to be the unique operator whose representing measure is equal to the restriction of $\mu$ to Borel subsets of $K_{E}^{\varepsilon}$ (see [10, Theorem VI.1.1]). Since $K_{E}^{\varepsilon}$ is Eberlein, the range of $\hat{T}$ is weakly compactly generated. Let $M \subseteq X$ be a convex, symmetric and weakly compact set such that

$$
\overline{\hat{T}\left(C\left(K_{E}^{\varepsilon}\right)\right)} \subset \bigcup_{n=1}^{\infty}\left(n M+\varepsilon B_{X}\right) .
$$

For every $f \in C(K)$ and $x^{*} \in X^{*},\left\|x^{*}\right\| \leqslant 1$, we have

$$
\left|x^{*}\left(T f-\left.\hat{T} f\right|_{K_{E}^{\varepsilon}}\right)\right|=\left|\int_{L^{\varepsilon}} f d\left(x^{*} \circ \mu\right)\right| \leqslant\left|x^{*} \circ \mu\right|\left(L^{\varepsilon}\right)\|f\| \leqslant \varepsilon\|f\| .
$$

This implies that

$$
T\left(B_{C(K)}\right) \subset \bigcup_{n=1}^{\infty}\left(n M+2 \varepsilon B_{X}\right)
$$

and, since ${\overline{n M+2 \varepsilon B_{X}}}^{w *} \subseteq X+2 \varepsilon B_{X^{* *}}$, each of the sets $n M+2 \varepsilon B_{X}$ is $2 \varepsilon$-weakly compact. Repeating this argument for $\varepsilon / k$ instead of $\varepsilon$ (for all $k \in \mathbb{N}$ ) we get a similar covering of $T\left(k B_{C(K)}\right)$ and, consequently, for some sequence $\left(M_{k}\right)_{k=1}^{\infty}$ of weakly compact sets we have $T(C(K)) \subset \bigcup_{k=1}^{\infty}\left(M_{k}+2 \varepsilon B_{X}\right)$, thus $\overline{T(C(K))}$ may be covered by countably many $3 \varepsilon$-weakly compact sets. It remains to appeal to Theorem 4.2 .

Now, we present two examples. The first one shows that the implication in Theorem 4.1 cannot be reversed, while the second one shows that the assumption of this theorem does 
not imply that there exists a decomposition $K=K_{E} \cup L$ with $K$ being Eberlein and $L$ being of semivariation zero.

Example 4.3. Consider a map $\varphi:\left[0, \omega_{1}\right] \rightarrow\left[0, \omega_{1}\right]$ given by

$$
\varphi(\alpha)= \begin{cases}\alpha+1, & \text { if } \alpha<\omega_{1} \text { is a successor ordinal, } \\ \alpha, & \text { if } \alpha \leqslant \omega_{1} \text { is a limit ordinal. }\end{cases}
$$

This is a continuous function, whence the composition operator $C_{\varphi}: C\left[0, \omega_{1}\right] \rightarrow C\left[0, \omega_{1}\right]$ defined by $C_{\varphi} f=f \circ \varphi$ is bounded. Now, put $T=I_{C\left[0, \omega_{1}\right]}-C_{\varphi}$.

Observe that $T$ maps the Schauder basis $\left(\mathbf{1}_{[0, \alpha]}\right)_{0 \leqslant \alpha \leqslant \omega_{1}}$ of $C\left[0, \omega_{1}\right]$ onto the set $\left\{e_{\alpha}\right\}_{\alpha \in D} \cup$ $\{0\}$, where $D$ is the set consisting of zero and all successors less than $\omega_{1}$. Hence, the range of $T$ is isometric to $c_{0}\left(\omega_{1}\right)$ which is a WCG Banach space. However, as we shall see, there is no decomposition $\left[0, \omega_{1}\right]=K \cup L$ with $K$ being Eberlein and with semivariation of $L$ less than 1.

Let $x^{*}=\left(x_{\alpha}\right)_{0 \leqslant \alpha \leqslant \omega_{1}} \in \ell_{1}\left[0, \omega_{1}\right]$ (identified with the dual space of $C\left[0, \omega_{1}\right]$ ). For each $f \in C\left[0, \omega_{1}\right]$ we have

$$
x^{*} T(f)=\sum_{\alpha \in D} x_{\alpha}(f(\alpha)-f(\alpha+1)),
$$

whence the representing measure for the functional $x^{*} T$ is given by

$$
\mu_{x^{*}}(\{\alpha\})= \begin{cases}x_{0}, & \text { if } \alpha=0 \\ x_{\alpha}-x_{\alpha^{\prime}}, & \text { if } \alpha=\alpha^{\prime}+1 \in D \\ 0, & \text { otherwise }\end{cases}
$$

Let $\mu$ stand for the representing measure for $T$. By the relation $\mu(A) x^{*}=\mu_{x^{*}}(A)$, for any Borel set $A \subseteq\left[0, \omega_{1}\right]$, and any $x^{*} \in C\left[0, \omega_{1}\right]^{*}$, we have

$$
\left|x^{*} \circ \mu\right|(A)=\sup _{\pi} \sum_{E_{j} \in \pi}\left|\mu\left(E_{j}\right) x^{*}\right|=\sum_{\alpha \in A}\left|\mu_{x^{*}}\{\alpha\}\right|,
$$

where $\pi$ is the set of all finite Borel partitions of $A$. Hence, whenever $A \cap D \neq \varnothing$, we have $\|\mu\|(A) \geqslant 1$. This shows that any decomposition $\left[0, \omega_{1}\right]=K \cup L$ with $\|\mu\|(L)<1$ would imply $D \subseteq K$, thus $K$ would be homeomorphic to the non-Eberlein space $\left[0, \omega_{1}\right]$.

Example 4.4. Define an operator $T: C(\beta \mathbb{N}) \cong \ell_{\infty} \rightarrow c_{0}$ by $T(\xi)=\left(\frac{1}{n} \xi_{n}\right)_{n=1}^{\infty}$. Then for each $x^{*}=\left(\eta_{n}\right)_{n=1}^{\infty} \in \ell_{1}$ the representing measure $\mu_{x^{*}}$ for $x^{*} T$ is supported on the set $\mathbb{N}$ and for each $n \in \mathbb{N}$ it takes the value $\frac{1}{n} \eta_{n}$. Then, similarly as above, we get $\left|x^{*} \circ \mu\right|(A)=\sum_{n \in A \cap \mathbb{N}} \frac{1}{n}\left|\eta_{n}\right|$ for every Borel set $A \subset \beta \mathbb{N}$. Therefore,

$$
\left.\|\mu\|(A)=\frac{1}{\min (A \cap \mathbb{N})} \quad \text { (with the convention } \frac{1}{\infty}=0\right) .
$$

Consequently, in order to have $\beta \mathbb{N}=K_{E}^{\varepsilon} \cup L^{\varepsilon}$ with $\|\mu\|\left(L^{\varepsilon}\right)<\varepsilon$, one should only guarantee that $\min \left(L^{\varepsilon} \cap \mathbb{N}\right)>\varepsilon^{-1}$. However, if we wish that $\beta \mathbb{N}=K \cup L$, where $K$ is Eberlein and $\|\mu\|(L)=0$, then necessarily $\mathbb{N} \subseteq K$, whence $K=\beta \mathbb{N}$ which is not Eberlein. 


\section{5. $\mathscr{W} \mathscr{C} \mathscr{G}(C(K))$ INSIDE $\mathscr{B}(C(K))$}

Let $K$ be a compact Hausdorff space. The 'magnitude' of $\mathscr{W} \mathscr{C} \mathscr{G}(C(K))$ in $\mathscr{B}(C(K))$ may be used as a naïve measure of the 'similarity of the space $K$ to an Eberlein compactum'. The first-named author together with Koszmider and Laustsen [17] proved that for $C\left[0, \omega_{1}\right]$ the ideal of weakly compactly generated operators is as big as possible, that is, it has codimension one in $\mathscr{B}\left(C\left[0, \omega_{1}\right]\right)$. On the other hand, Laustsen and Loy [23, p. 253] noticed, based on well-known facts, that the ideal of weakly compact operators on $\ell_{\infty}=C(\beta \mathbb{N})$ is the unique maximal ideal of $\mathscr{B}\left(\ell_{\infty}\right)$. The space $\ell_{\infty}$ is Grothendieck [9, Theorem VII.15], hence it follows from [8, Corollary 5, p. 150] that $\mathscr{W}\left(\ell_{\infty}\right)=\mathscr{W} \mathscr{C} \mathscr{G}\left(\ell_{\infty}\right)$. However, the codimension of $\mathscr{W} \mathscr{C} \mathscr{G}\left(\ell_{\infty}\right)$ in $\mathscr{B}\left(\ell_{\infty}\right)$ is infinite, as can be easily seen.

We shall add to this picture a $C(K)$-space constructed by Koszmider [19] for which the ideal of weakly compactly generated operators has codimesion one in $\mathscr{B}(C(K))$. This space has an interesting feature: we are able to give a complete description of the lattice of closed ideals of $\mathscr{B}(C(K))$ what we shall do. The first-named author was informed by P.A.H. Brooker that he also obtained a similar result (unpublished) independently.

Theorem 5.1 (Koszmider [19]). Assuming the continuum hypothesis $\mathrm{CH}$ or Martin's Axiom with $\neg \mathrm{CH}$, there exists a compact scattered Hausdorff space $K$ such that:

(i) the ideal $\mathscr{X}(C(K))$ has codimension one in $\mathscr{B}(C(K))$;

(ii) each separable subspace of $C(K)$ is contained in a subspace isomorphic to $c_{0}$;

(iii) if $C(K)=A \oplus B$ is a decomposition into two closed, infinite-dimensional subspaces $A$ and $B$, then either $A \cong c_{0}$ and $B \cong C(K)$ or vice versa

Remark 5.2. The above-mentioned space $K$ is a special example of a Mrówka space, that is, the Stone space of the Boolean subalgebra of $\mathscr{P}(\mathbb{N})$ generated by an uncountable family of almost disjoint sets together with all finite subsets of $\mathbb{N}$. Mrówka spaces are classical examples of scattered compacta which are not Eberlein. Consequently, the ideal $\mathscr{W} \mathscr{C} \mathscr{G}(C(K))$ is properly contained in $\mathscr{B}(C(K))$. Since $\mathscr{W} \mathscr{C} \mathscr{G}(C(K))$ contains the maximal ideal $\mathscr{X}(C(K))$ and is proper, we have $\mathscr{W} \mathscr{C} \mathscr{G}(C(K))=\mathscr{X}(C(K))$.

For this particular space $K$ we can describe the lattice of all closed ideal in $\mathscr{B}(C(K))$. To do that we need to gather some well-known facts. Each Radon measure on a compact scattered space $K$ is countably supported, whence there is a natural isometric identification between the dual space of $C(K)$ and the Banach space $\ell_{1}(K)$. In particular, the dual space $C(K)^{*}$ enjoys the Schur property. Moreover, we shall require the following theorem due to Lotz, Peck and Porta [25]:

Theorem 5.3. Let $K$ be a compact Hausdorff space. Then, $K$ is scattered if and only if each closed, infinite-dimensional subspace of $C(K)$ contains a subspace which is isomorphic to $c_{0}$ and complemented in $C(K)$.

Proposition 5.4. Let $K$ be a compact scattered Hausdorff space. Then,

(i) $\mathscr{K}(C(K))=\mathscr{W}(C(K))$;

(ii) no closed ideal lies between $\{0\}$ and $\mathscr{K}(C(K))$ or $\mathscr{K}(C(K))$ and $\mathscr{G}_{c_{0}}(C(L))$. 
Proof. Part (i) is standard: the dual space $C(K)^{*}=\ell_{1}(K)$ has the Schur property, whence $\mathscr{K}\left(\ell_{1}(K)\right)=\mathscr{W}\left(\ell_{1}(K)\right)$. The claim follows from Gantmacher's theorem and Schauder's theorem.

To prove part (ii) let us notice that the space $C(K)$, being an $\mathscr{L}_{\infty}$-space, has the bounded approximation property, hence the ideal of compact operators $\mathscr{K}(C(K))$ is the smallest closed non-trivial ideal in $\mathscr{B}(C(K))$. It remains to show that if $T \in \mathscr{B}(C(K)) \backslash \mathscr{W}(C(K))$ then the ideal generated by $T$ contains the ideal $\mathscr{G}_{c_{0}}(C(K))$. By a result of Pełczyński (cf. [28] or [10, Theorem VI.2.15]), there is a subspace $Y$ of $C(K)$ isomorphic to $c_{0}$ such that $\left.T\right|_{Y}$ is bounded below. Hence $T(Y)$ is closed, so by Theorem 5.3, it contains a completemented copy of $c_{0}$, say $Z$. Note that $\left.T\right|_{X}: X \rightarrow Z$ is an isomorphism which factors the identity operator on $c_{0}$. Consequently, $\mathscr{G}_{c_{0}}(C(K))$ is contained in the ideal generated by $T$.

Theorem 5.5. Let $K$ be the Mrówka space constructed by Koszmider in [19]. Then the the lattice of closed ideals in $\mathscr{B}(C(K))$ has the form:

$$
\{0\} \subsetneq \mathscr{K}(C(K)) \subsetneq \mathscr{X}(C(K))=\mathscr{G}_{c_{0}}(C(K))=\mathscr{W} \mathscr{C} \mathscr{G}(C(K)) \subsetneq \mathscr{B}(C(K)) .
$$

Proof of Theorem 5.5. Since the space $K$ is scattered, no closed ideal lies neither between $\{0\}$ and $\mathscr{K}(C(K))$ nor $\mathscr{K}(C(K))$ and $\mathscr{G}_{c_{0}}(C(K))$ (Proposition 5.4(iii)). By Remark 5.2, we have $\mathscr{W} \mathscr{C} \mathscr{G}(C(K))=\mathscr{X}(C(K))$; let $T \in \mathscr{X}(C(K))$. By Theorem 5.1(b), there is a subspace $Y$ isomorphic to $c_{0}$ such that $T(C(K)) \subseteq Y$, so $T$ factors through $c_{0}$. Thus, the ideals $\mathscr{G}_{c_{0}}(C(K))$ and $\mathscr{X}(C(K))$ are equal by virtue of the maximality of the latter one. Now, if $\mathscr{J}$ is any maximal ideal in $\mathscr{B}(C(K))$, by Proposition 5.4(ii), it must contain $\mathscr{G}_{c_{0}}(C(K))$, hence $\mathscr{J}=\mathscr{G}_{c_{0}}(C(K))$, thus there are no other closed ideals in $\mathscr{B}(C(K))$ than those listed in the claim.

\section{REFERENCES}

1. D. Alspach and Y. Benyamini, Primariness of spaces of continuous functions on ordinals, Israel J. Math. 27 (1977), 64-92.

2. D. Amir and J. Lindenstrauss, The structure of weakly compact sets in Banach spaces, Ann. of Math. 88 (1968), 35-46.

3. S.A. Argyros and S. Mercourakis, Examples concerning heredity problems of WCG Banach spaces, Proc. Amer. Math. Soc. 133 (2005), 773-785.

4. B. Blackadar, K-Theory for Operator Algebras, $2^{\text {nd }}$ edition, Math. Sci. Res. Inst. Publ., vol. 5, Cambridge Univ. Press, Cambridge 1998.

5. P.G. Casazza, B.-L. Lin and R.H. Lohman, On James' quasi-reflexive Banach space, Proc. Amer. Math. Soc. 67 (1977), 265-271.

6. J. Bourgain and J. Diestel, Limited operators and strict co-singularity. Math. Nachr., 119 (1984), $55-58$.

7. J. Diestel, Grothendieck spaces and vector measures, in: Vector and Operator Valued Measures and Applications, Academic Press (1973), pp. 97-108.

8. J. Diestel, Geometry of Banach Spaces - Selected Topics, Lecture Notes in Math. 485, SpringerVerlag 1975.

9. J. Diestel, Sequences and Series in Banach Spaces, Springer-Verlag, New York, 1984.

10. J. Diestel and J.J. Uhl, Vector Measures. With a foreword by B.J. Pettis, Mathematical Surveys, no. 15, American Mathematical Society, Providence, R.I. 1977.

11. D. Dosev and W.B. Johnson, Commutators on $\ell_{\infty}$, Bull. London Math. Soc. 42 (2010), $155-169$. 
12. G.A. Edgar, A long James space, in: Measure Theory, Oberwolfach 1979, Lectures Notes in Math. 794, Springer-Verlag (1980), pp. 31-37.

13. M. Fabian, V. Montesinos and V. Zizler, A characterization of subspaces of weakly compactly generated Banach spaces, J. London Math. Soc. 69 (2004) 457-464.

14. J. Hagler and W.B. Johnson, On Banach spaces whose dual balls are not weak* sequentially compact, Israel J. Math. 28 (1997), 325-330.

15. P. Hájek, V. Montesinos, J. Vanderwerff and V. Zizler, Biorthogonal Systems in Banach Spaces, CMS Books in Mathematics, Springer-Verlag 2008.

16. W.B. Johnson and J. Lindenstrauss, Some remarks on weakly compactly generated Banach spaces, Israel J. Math. 17 (1974), 219-230.

17. T. Kania, P. Koszmider and N.J. Laustsen, A weak*-topological dichotomy with applications in operator theory, Trans. London Math. Soc. (to appear).

18. T. Kania and N.J. Laustsen, Uniqueness of the maximal ideal of the Banach algebra of bounded operators on $C\left(\left[0, \omega_{1}\right]\right)$, J. Funct. Anal., 262, 4831-4850.

19. P. Koszmider, On decompositions of Banach spaces of continuous functions on Mrówka's spaces, Proc. Amer. Math. Soc. 133 (2005), 2137-2146.

20. N.J. Laustsen, K-Theory for the Banach algebra of operators on James's quasi-reflexive Banach spaces, K-Theory 23 (2000), 115-127.

21. N.J. Laustsen, Maximal ideals in the algebra of operators on certain Banach spaces, Proc. Edinb. Math. Soc. 45 (2002), 523-546.

22. N.J. Laustsen, Commutators of operators on Banach spaces, J. Operator Theory 48 (2002), 503-514.

23. N.J. Laustsen and R.J. Loy, Closed ideals in the Banach algebra of operators on a Banach space, Proceedings of the Conference on Topological Algebras, their Applications, and Related Topics (ed. K. Jarosz and A. Soltysiak), Banach Center Publications 67 (2005), 245-264.

24. R.J. Loy and G.A. Willis, Continuity of derivations on $B(E)$ for certain Banach spaces E, J. London Math. Soc. 40 (1989), 327-346.

25. H.P. Lotz, N.T. Peck and H. Porta, Semi-embeddings of Banach spaces, Proc. Edinb. Math. Soc., II. Ser. 22 (1979), 233-240.

26. J. Lindenstrauss, Weakly compact sets: their topological properties and the Banach spaces they generate, R.D. Anderson (ed.), Symp. Infinite Dimensional Topol., Math. Studies 69 (1972), pp. 235-273.

27. J. Lindenstrauss and L. Tzafriri, Classical Banach Spaces II, Springer-Verlag 1979.

28. A. Pełczyński, Banach spaces on which every unconditionally converging operator is weakly compact, Bull. Acad. Pol. Sci. Ser. Math. Astr. Phys. 10 (1962), 265-270.

29. A. Pietsch, Operator Ideals, North-Holland, Amsterdam 1980.

30. H.P. Rosenthal, The heredity property for weakly compactly generated Banach spaces, Compositio Math. 2 (1974), 83-111.

31. G.A. Willis, Compressible operators and the continuity of homomorphisms from algebras of operators, Studia Math. 115 (1995), 251-259.

Department of Mathematics and Statistics, Fylde College, Lancaster University, LanCaster LA1 4YF, United Kingdom and Institute of Mathematics, Polish Academy of SciENCES, ŚniadeCKich 8, 00-956 WarszaWa, Poland

E-mail address: t.kania@lancaster.ac.uk

Institute of Mathematics, Polish Academy of Sciences, Śniadeckich 8, 00-956 Warszawa, Poland and Institute of Mathematics, University of Silesia, Bankowa 14, 40-007 Katowice, POLAND

E-mail address: t_kochanek@wp.pl 